\title{
TRAUMA DO PÂNCREAS: fatores preditivos de morbidade e mortalidade relacionados a índices de trauma
}

\author{
Henrique José Virgili SILVEIRA, Mario MANTOVANI e Gustavo Pereira FRAGA
}

RESUMO - Contexto - Embora incomuns, lesões traumáticas do pâncreas estão associadas a significativos níveis de complicações e mortalidade. Objetivo - Definir os fatores preditivos de morbidade e mortalidade em pacientes vítimas de trauma pancreático. Método - Foram estudados 131 pacientes atendidos pela Disciplina de Cirurgia do Trauma no Hospital de Clínicas da Universidade Estadual de Campinas, SP, no período entre janeiro de 1994 a dezembro de 2007, com seus parâmetros epidemiológicos, fisiológicos e anatômicos, sendo comparados e analisados aos fatores preditivos de evolução, com estudo estatístico. Resultados - Trauma penetrante, com predomínio de ferimentos por projétil de arma de fogo ocorreu em $64 \%$ dos casos. A maioria, 91,6\%, era do sexo masculino e a idade média de 29,8 anos. A morbidade global foi de 64,9\%, com $29 \%$ de complicações diretamente relacionadas ao pâncreas, como fístulas e sangramento. A mortalidade foi de 27,5\%, principalmente em decorrência de choque hipovolêmico e falência de múltiplos órgãos e sistemas. Houve maior morbidade e mortalidade em pacientes com lesões complexas (graus IV e V) do pâncreas quando comparadas com lesões menos graves (graus I e II), porém a morbidade e mortalidade neste grupo não foram desprezíveis, devido a valores alterados de escore de trauma revisado ("revised trauma score"), valores elevados de índice de gravidade da lesão ("injury severity score") e "abdominal trauma index". Conclusões - Valores alterados de "revised trauma score", pressão arterial sistólica menor que $90 \mathrm{~mm} \mathrm{Hg}$, valor de "injury severity score" menor ou igual a 15 e valor de "abdominal trauma index" maior ou igual a 25 são fatores relacionados a aumento da morbidade. Valores anormais de "revised trauma score", valores de "injury severity score" e "abdominal trauma index" superiores a 25, pressão arterial sistólica inferior a $90 \mathrm{~mm} \mathrm{Hg}$ são fatores preditivos de aumento de mortalidade em pacientes vítimas de trauma pancreático. Valores baixos do índice misto, anatômico e fisiológico denominado TRISS, são preditivos de maior morbidade e mortalidade, porém valores elevados deste índice não são preditivos de boa evolução. DESCRITORES - Pâncreas, lesões. Índices de gravidade do trauma.

\section{INTRODUÇÃO}

As principais causas de morte entre indivíduos com idade até 35 anos são externas, resultantes do aumento contínuo da violência. $\mathrm{O}$ abdome é frequentemente acometido tanto por lesões penetrantes quanto por traumas fechados ${ }^{(14,22,23)}$. O pâncreas é um órgão de localização retroperitonial, na porção central do andar superior do abdome, medindo de 15 a $20 \mathrm{~cm}$ de comprimento e com massa de 80 a 90 g. Devido a sua proximidade com outras estruturas, tais como aorta, veia cava, veia porta, vasos mesentéricos, estômago, cólon e sua íntima relação com o duodeno, lesões isoladas do pâncreas são pouco frequentes, ocorrendo em apenas $2 \%$ dos traumatismos abdominais, sendo decorrentes em dois terços das vezes de traumas penetrantes e em um terço de traumas abdominais fechados. Em casos de trauma abdominal fechado, devido a sua proximidade com a coluna vertebral, o pâncreas é lesado entre 3\% e $12 \%$ das vezes ${ }^{(17,19,21,25,26,28,)}$.
Cerca de $75 \%$ das lesões pancreáticas são simples, constituindo-se apenas em hematoma, lesão da cápsula ou lesão do parênquima, sem acometimento do ducto pancreático principal ${ }^{(19)}$.

A mortalidade das lesões pancreáticas, ao contrário de sua baixa incidência, é elevada, atingindo acima de $50 \%$ dos casos onde há lesão complexa do órgão $0^{(19,20,24,}$ ${ }^{30)}$. O óbito ocorre em até $75 \%$ das vezes nas primeiras 48 horas após o trauma, decorrente de hemorragia. Infecção e falência de múltiplos órgãos são responsáveis pelos demais casos de óbito ${ }^{(1,3)}$. Lesões associadas de pâncreas e duodeno compreendem menos de 10\% das lesões pancreáticas, porém estão associadas a maior índice de complicações e mortalidade ${ }^{(19,26)}$. Devido à íntima relação anatômica entre pâncreas e duodeno, o mesmo procedimento pode ser utilizado para tratamento de ambas as lesões, bem como para minimizar as complicações pós-operatórias ${ }^{(15,18)}$.

Para se avaliar a gravidade de um trauma, foram desenvolvidos diversos índices, de modo a mensurar a

Trabalho realizado na Disciplina de Cirurgia do Trauma, Departamento de Cirurgia da Faculdade de Ciências Médicas da Universidade Estadual de Campinas (UNICAMP), Campinas, SP.

Correspondência: Dr. Henrique José Virgili Silveira - Rua Dr. Arthur Almeida Resende, 98 - 13083-150 - Campinas, SP. E-mail: henriquejvs@gmail.com 
gravidade anatômica das lesões, bem como sua repercussão sistêmica no indivíduo traumatizado ${ }^{(11)}$.

O escore de trauma revisado ("Revised Trauma Score" ou RTS), utiliza para seu cálculo a escala de coma de Glasgow (ECG), a pressão arterial sistólica (PAS) e a frequência respiratória (FR), com cada parâmetro recebendo valor de 0 a 4 aproximados, de acordo com a probabilidade de sobrevida em cada um deles. Cada parâmetro recebeu peso diferente, compondo a fórmula: $\mathrm{RTS}=0,9368 \times \mathrm{ECG}+0,7326 \times \mathrm{PAS}$ $+0,2908 \times$ FR. Os valores do RTS variam de 0 a 7,84 e devem ser calculados à admissão do paciente no serviço $\operatorname{médico}^{(9,10)}$.

A escala de gravidade da lesão pancreática ("organ injury scale" ou OIS) classifica as lesões anatômicas do órgão em graus, de forma a padronizar sua descrição e permitir análise comparativa, conforme mostrado na Figura $1^{(23)}$.

\begin{tabular}{l}
\begin{tabular}{l|ll|}
\hline Grau I & $\bullet$ & Contusão ou laceração menor, sem envolver ducto principal \\
\hline Grau II & $\bullet$ & Transecção distal ou laceração distal com lesão ductal \\
\hline Grau III & $\bullet$ & Transecção proximal ou lesão do parênquima com provável lesão ductal \\
\hline Grau IV & $\bullet$ & Lesão combinada de pâncreas e duodeno, com papila e vascularização preservadas \\
\hline Grau V & $\bullet$ & Lesão maciça, lesão da papila e desvascularização \\
* Moore EE et al. ${ }^{22)}$ \\
FIGURA 1. Classificação das lesões do pâncreas*
\end{tabular} \\
\hline
\end{tabular}

O índice de gravidade da lesão ("injury severity score" ou ISS) é utilizado para quantificar a gravidade das lesões em pacientes politraumatizados. Este índice divide o corpo humano em seis segmentos: cabeça e pescoço, face, tórax, abdome e órgãos pélvicos, extremidades e ossos da pelve, e superfície externa. Em cada segmento cada lesão recebe uma pontuação de 1 a 6 , segundo os seguintes critérios: 1, representa lesão menor, 2 , lesão moderada, 3 , lesão maior ou grave, 4, lesão severa, 5, lesão crítica e 6 , lesão fatal. Considera-se apenas a lesão mais grave em cada segmento. A seguir, tomam-se os três segmentos que apresentaram os maiores valores, excluindo-se os demais segmentos, eleva-se cada um desses três valores ao quadrado e somam-se os três quadrados, obtendo o valor do ISS. Os valores variam de 0 a 75, com a mortalidade sendo diretamente proporcional ao aumento deste valor ${ }^{(4)}$.

O índice de trauma abdominal ("abdominal trauma index" ou ATI) avalia trauma abdominal penetrante e fechado. Cada órgão abdominal tem um fator de risco que varia de 1 a 5 e, em cada órgão a lesão recebe pontos de acordo com sua gravidade, também de 1 a 5 . O escore de lesão de cada órgão é o produto do fator de risco pela gravidade da lesão neste órgão e a soma dos escores de todos os órgãos lesados representa o ATI. A vantagem deste índice é considerar todas as lesões intra-abdominais, independente de sua gravidade, porém não considera lesões em outros segmentos do corpo que também afetam a morbimortalidade. Pacientes que apresentam ATI maior que 25 têm elevado risco de desenvolver complicações e evoluir para óbito ${ }^{(6,22)}$.

Utilizando os resultados obtidos no RTS e no ISS, a idade do paciente e o tipo de trauma, penetrante ou fechado, pode- se calcular o TRISS que é um índice misto, calculado a partir do ISS e do RTS, daí sua denominação. Esses valores são aplicados a uma tabela, denominada TRISSCAN, utilizando os valores do ISS, do RTS, a idade do paciente e o tipo de trauma (penetrante ou contuso) que, então, determinará a probabilidade de sobrevida e sua significância ${ }^{(7)}$.

Apesar da baixa incidência do trauma de pâncreas, a dificuldade diagnóstica associada a altas taxas de morbimortalidade evidencia a importância do conhecimento do cirurgião a respeito das vicissitudes que envolvem pacientes com lesão pancreática. Com isso, este estudo teve como objetivo a avaliação e análise dos pacientes vítimas de trauma de pâncreas atendidos pela Disciplina de Cirurgia do Trauma da Universidade Estadual de Campinas (UNICAMP), Campinas, SP, visando identificar as complicações gerais e as diretamente relacionadas ao procedimento, bem como o índice de mortalidade, a fim de definir dentre as variáveis estudadas os fatores preditivos de maior morbimortalidade relacionados a esta lesão.

\section{MÉTODO}

Estudo coorte prospectivo de casos registrados em protocolo da Disciplina de Cirurgia do Trauma do Departamento de Cirurgia da Faculdade de Ciências Médicas (FCM) da UNICAMP. Os sujeitos da pesquisa foram os pacientes atendidos e tratados, no período de 1994 a 2007 por lesão de pâncreas, excluindo os pacientes com idade inferior a 14 anos, com lesão pancreática e operados em outros serviços encaminhados posteriormente, ou pacientes com lesões iatrogênicas. O projeto do estudo foi aprovado pelo Comitê de Ética em Pesquisa da FCM-UNICAMP, conforme o parecer $n^{\circ} 627 / 2008$.

Para cada um dos pacientes com lesão do pâncreas foi aplicado um protocolo, em que constam as seguintes informações: idade, sexo, tipo de traumatismo, sinais e sintomas na admissão, propedêutica complementar realizada, tempo entre trauma e cirurgia, tempo entre admissão e cirurgia, localização da lesão, grau da lesão, presença de sangramento na cavidade, contaminação da cavidade, procedimento cirúrgico realizado, tipos e graus de lesões associadas, o RTS, o ATI, o ISS e TRISS, as complicações (locais e sistêmicas), realização ou não de relaparotomia ou outras cirurgias, dias de internação e a evolução do paciente (alta ou óbito).

Os resultados foram analisados através do software Epi-Info versão 6.04. Foram empregados o teste do qui ao quadrado ou teste exato de Fisher. O nível de significância foi definido como $P<0,05$. O valor de corte dos índices referidos anteriormente foi definido como aquele em que passou a haver significância estatística entre os grupos, acima e abaixo deste valor.

As lesões pancreáticas foram classificadas conforme estabelecido pela Associação Americana de Cirurgia do Trauma (AAST), exposto na Figura 1. Para o tratamento da lesão pancreática foram consideradas as seguintes condutas: 
drenagem, sutura, pancreatectomia distal com ou sem esplenectomia, e gastroduodenopancreatectomia.

\section{RESULTADOS}

No período entre janeiro de 1994 e dezembro de 2007 foram realizadas 2.778 cirurgias em vítimas de trauma, considerandose apenas casos novos, sendo excluídas as reoperações, pela equipe da Disciplina de Cirurgia do Trauma da UNICAMP, das quais, 2.109 foram laparotomias exploratórias. Deste total, 131 pacientes apresentavam trauma pancreático e foram tratados cirurgicamente. Os doentes eram do sexo masculino em 120 casos $(91,6 \%)$ e a sua idade variou entre 15 e 69 anos, com média de 29,8 anos.

Oitenta e seis foram vítimas de trauma penetrante $(65,7 \%)$, em sua maioria por projétil de arma de fogo (59 casos, $68,6 \%$ ) e outros $27(31,4 \%)$ vítimas de ferimento por arma branca. Quarenta e cinco pacientes foram vítimas de trauma abdominal fechado, sendo 20 por acidente automobilístico (44,4\%), 9 por atropelamento $(20 \%), 5$ por acidente com motocicleta $(11,1 \%), 5$ por espancamento e 3 casos de trauma por animais $(6,7 \%), 2$ por quedas de altura $(4,4 \%)$ e 1 por acidente com bicicleta $(2,2 \%)$.

No grupo de pacientes vítimas de trauma penetrante, 61 $(70,9 \%)$ apresentaram complicações pós-operatórias e 27 evoluíram para óbito $(31,4 \%)$. Dentre os pacientes vítimas de trauma abdominal contuso, $24(53,3 \%)$ apresentaram complicações pós-operatórias e 9 foram a óbito $(20 \%)$. Comparando os dois grupos entre si, não se observou diferença estatisticamente significante com relação à mortalidade $(P=$ $0,23)$, porém houve tendência a maior morbidade no grupo vítima de trauma penetrante $(P=0,07)$.

Todos os pacientes vítimas de trauma penetrante foram submetidos a laparotomia. Aqueles vítimas de trauma contuso foram submetidos a propedêutica complementar com tomografia computadorizada realizada em 14 casos $(51,9 \%)$, identificando lesão pancreática em 11 casos $(78,6 \%)$.

O intervalo entre o trauma e a cirurgia foi inferior a 6 horas em todos os pacientes com lesão penetrante e em $47 \%$ dos casos de trauma contuso, conforme mostrado na Tabela 1.

TABELA 1. Número de casos conforme o intervalo de tempo transcorrido entre o trauma e a cirurgia

\begin{tabular}{lcc}
\hline Intervalo de tempo & $\begin{array}{c}\text { Trauma penetrante } \\
(\mathrm{n}=86 \text { casos }-65,7 \%)\end{array}$ & $\begin{array}{c}\text { Trauma fechado } \\
(\mathrm{n}=45 \text { casos }-34,3 \%)\end{array}$ \\
\hline Até 2 horas & $52(60,5 \%)$ & $4(8,9 \%)$ \\
2 a 6 horas & $34(39,5 \%)$ & $19(42,2 \%)$ \\
6 a 12 horas & - & $13(28,9 \%)$ \\
12 a 24 horas & - & $7(15,6 \%)$ \\
Maior que 24 horas & - & $2(4,4 \%)$ \\
\hline
\end{tabular}

Cinquenta e um pacientes apresentaram lesão grau I $(38,9 \%)$, 30 apresentaram lesão grau II (22,9\%), 36 lesão grau III (27,5\%), 9 lesão grau IV (6,9\%) e 5 lesão grau V(3,8\%) (Figura 2).

Em 118 pacientes $(90,1 \%)$ foram observadas lesões de outros órgãos associadas à lesão pancreática. $\mathrm{O}$ órgão mais acometido foi o fígado, em 58 casos, seguido pelo estômago

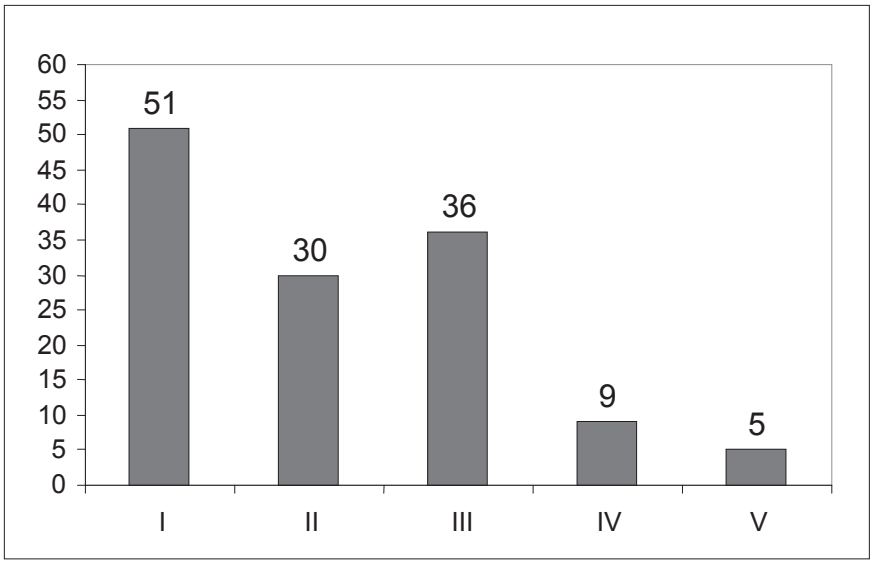

FIGURA 2. Número de casos conforme o grau de lesão do pâncreas

em 54 e duodeno em 33. A lesão duodenal foi grau I em 1 caso, grau II em 13, grau III em 8, grau IV em 7 e grau V em 4. Ocorreu lesão de cólon e/ou reto em 34 pacientes e de delgado em 21. Lesão de grandes vasos abdominais foi observada em 39 pacientes, sendo 12 de veia cava e 3 de aorta. Todas as lesões associadas à lesão pancreática estão apresentadas na Figura 3

\begin{tabular}{|l|c|}
\hline Órgãos/vísceras lesados & Número de casos \\
\hline Fígado & 58 \\
\hline Estômago & 54 \\
\hline Cólon e reto & 34 \\
\hline Duodeno & 33 \\
\hline Baço & 27 \\
\hline Rim & 27 \\
\hline Diafragma & 24 \\
\hline Grandes vasos abdominais & 24 \\
\hline Jejuno-íleo & 21 \\
\hline Veia cava & 12 \\
\hline Vesícula/via biliar & 6 \\
\hline Pequenos vasos abdominais & 4 \\
\hline Aorta & 3 \\
\hline Bexiga & 3 \\
\hline Outras & 14 \\
\hline
\end{tabular}

FIGURA 3. Número de casos conforme a presença de lesões associadas em outros órgãos e estruturas vasculares

O período de internação variou de 3 a 68 dias, com média de 17 dias e mediana de 12 dias, considerando-se apenas os pacientes que sobreviveram à cirurgia. Quinze pacientes $(11,5 \%)$ evoluíram a óbito nas primeiras horas após o procedimento cirúrgico, não completando 1 dia de internação. Destes, seis foram a óbito na sala de cirurgia, não sobrevivendo ao procedimento cirúrgico. Outros sete $(5,3 \%)$ pacientes foram a óbito nas primeiras 72 horas após a cirurgia.

A técnica cirúrgica mais utilizada foi apenas drenagem em 46 casos $(35,1 \%)$, seguida de pancreatectomia distal, que foi realizada em 39 pacientes $(29,8 \%)$, sendo 8 casos de pancreatectomia caudal e 31 de corpo-caudal. Foi realizada preservação esplênica em $10(25,6 \%)$ destes casos. 
Apenas sutura do pâncreas foi realizada em 36 pacientes $(27,5 \%)$. Quatro deles com lesão grau V foram submetidos a gastroduodenopancreatectomia (3\%). Esses procedimentos estão apresentados na Figura 4. Todos os pacientes foram mantidos com drenagem a vácuo após o procedimento. Seis $(4,6 \%)$ foram a óbito durante o procedimento cirúrgico, sem que este fosse concluído, devido a choque hemorrágico.



FIGURA 4. Número de cirurgias realizadas nos casos de trauma de pâncreas

A morbidade global foi de $64,9 \%$, envolvendo 85 pacientes e a mortalidade $27,5 \%$, com 36 pacientes evoluindo a óbito.

Complicações relacionadas diretamente à lesão pancreática foram observadas em 38 pacientes $(29 \%)$, sendo a mais frequente fístula pancreática, encontrada em 23 casos (17,6\%), sendo ainda encontrados 16 casos de pancreatite aguda $(12,2 \%), 7$ abscessos intracavitários $(5,3 \%)$ e 3 pacientes que apresentaram ressangramento abdominal com origem no pâncreas $(2,3 \%)$.

Complicações gerais não relacionadas à lesão pancreática ou ao procedimento cirúrgico no pâncreas ocorreram em 82 pacientes $(62,6 \%)$, com predomínio de sangramento de outros sítios que não o pâncreas em 25 casos $(19,1 \%)$, complicações pulmonares em 19 casos $(14,5 \%)$, sepse e falência de múltiplos órgãos em 18 casos $(13,7 \%)$. Foram ainda foram observados 10 pacientes com fístulas do trato gastrointestinal $(7,6 \%), 9$ com insuficiência renal $(6,9 \%), 7$ com infecção de ferida operatória (5,3\%), 7 com complicações neurológicas secundárias a trauma craniencefálico (TCE) e 3 com infecção do trato urinário $(2,3 \%)$.

O índice de mortalidade foi de $27,5 \%$, com 36 pacientes evoluindo a óbito. A causa mais frequente deste foi choque hemorrágico, observada em 19 casos (52,8\%). Outros 13 pacientes foram a óbito em decorrência de sepse e falência de múltiplos órgãos e sistemas e 4 devido a complicações neurológicas secundárias a trauma craniencefálico.

Oito pacientes com lesão grau I (15,7\%), 9 com lesão grau II (30\%), 18 com lesão grau III (50\%), 2 com lesão grau IV $(22,2 \%)$ e 3 com lesão grau V $(60 \%)$ apresentaram morbidade pós-operatória.

Nos pacientes com lesão grau I, houve 7 óbitos (13,7\%), no grupo com lesão grau II, 10 óbitos $(33,3 \%)$, nos casos de lesão grau III também 10 óbitos $(27,8 \%)$, nos casos de lesão grau IV 6 óbitos $(66,7 \%)$ e 3 nos casos de lesão grau V (60\%).
Comparando-se a incidência de morbidade e mortalidade por grau de lesão, observou-se que as lesões grau I e II apresentaram menor morbidade e mortalidade, quando comparadas com grau IV e V, com diferença estatisticamente significante $(P=0,003)$.

Os valores de RTS variaram entre 0,29 e o máximo, valor considerado normal de 7,84, com média igual a 7,0. Este índice apresentou diferença estatisticamente significante com todos os valores alterados, quando comparados ao grupo com valor máximo $(7,84)$, considerado normal. $\mathrm{O}$ valor de RTS foi normal em 87 casos $(66,4 \%)$ e destes, $46(52,9 \%)$ apresentaram alguma complicação mórbida. Outros 44 casos $(33,6 \%)$ apresentaram valor alterado para este índice, com 39 apresentando complicações $(88,6 \%)$. No grupo com valor de RTS normal, houve 9 óbitos (10,3\%) e no outro grupo 27 $(61,4 \%)$. Houve diferença estatisticamente significante quando comparados os grupos de valores normais e alterados de RTS, tanto para morbidade quanto para mortalidade, com valores de $P<0,0001$ (Figura 5).

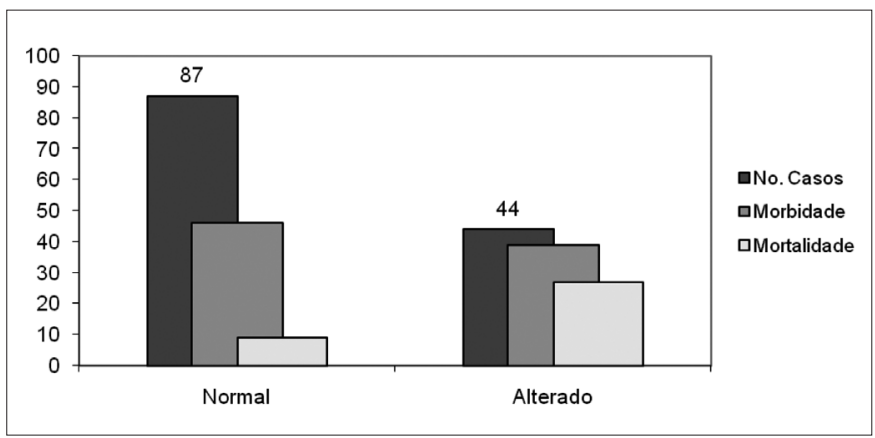

FIGURA 5. Relação entre RTS, morbidade e mortalidade

Observaram-se 34 pacientes (26\%) admitidos no serviço com PAS inferior a $90 \mathrm{~mm} \mathrm{Hg}$ e outros 97 (74\%) casos com PAS superior ou igual a $90 \mathrm{~mm} \mathrm{Hg}$.

No primeiro grupo de pacientes, considerados em choque, observaram-se 29 complicações pós-operatórias $(85,3 \%$ e e no segundo grupo, 56 casos com complicações pós-operatórias $(57,7 \%)$. Houve diferença significante estatisticamente entre os dois grupos $(P=0,007)$.

Com relação à mortalidade, houve 21 óbitos no grupo de pacientes com PAS inferior a $90 \mathrm{~mm} \mathrm{Hg}(61,8 \%)$ e 15 no grupo com PAS superior ou igual a $90 \mathrm{~mm} \mathrm{Hg}(15,4 \%)$. Comparando-se os dois grupos, foi encontrada diferença estatisticamente significante entre eles $(P<0,0001)$. A relação de morbidade e mortalidade com a PAS no momento da admissão do paciente é mostrada na Tabela 2.

O ISS variou de 4 a 66 , com média de 20,7 , sendo que 95 casos $(72,5 \%)$ apresentaram valor inferior ou igual a 25 . Dentre os pacientes com valor de ISS menor ou igual a $25,59(92,1 \%)$ apresentaram complicações, com 19 indo a óbito $(20 \%)$. No grupo de pacientes com valor acima de 25 , observaram-se 26 pacientes com complicações pós-operatórias $(72,2 \%)$ e 17 óbitos $(47,2 \%)$. Não houve diferença significante 
entre os dois grupos com relação à morbidade $(P=0,77)$, porém com relação à mortalidade foi observada diferença estatística significante $(P=0,003)$ (Figura 6).

TABELA 2. Relação entre PAS, morbidade e mortalidade

\begin{tabular}{lccc}
\hline PAS & $\mathbf{N}^{\circ}$ de casos & Morbidade & Mortalidade \\
\hline$>90 \mathrm{~mm} \mathrm{Hg}$ & $97(74 \%)$ & $56(57,7 \%)$ & $15(15,4 \%)$ \\
$<=90 \mathrm{~mm} \mathrm{Hg}$ & $34(26 \%)$ & $29(85,3 \%)$ & $21(61,8 \%)$ \\
& & $P=0,007$ & $P<0,0001$ \\
\hline
\end{tabular}

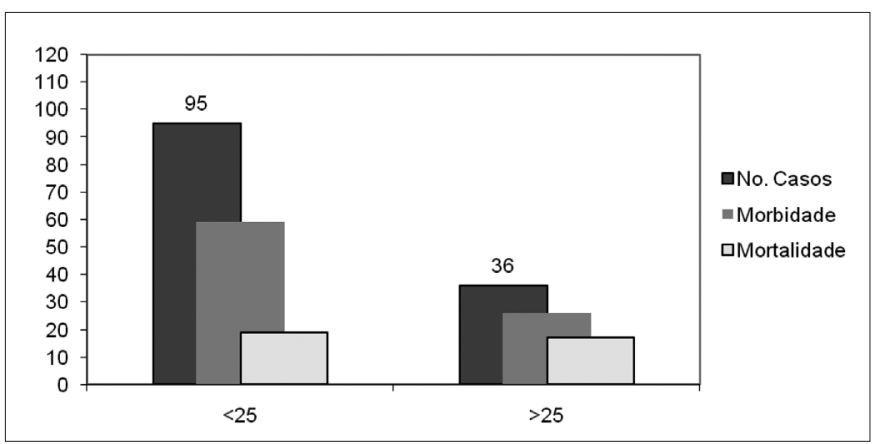

FIGURA 6. ISS relacionado com morbidade e mortalidade (valor de corte do ISS $=25$ )

Realizando a mesma comparação com valores de ISS inferiores ou iguais a 15 e superiores a 15, encontraram-se 37 pacientes no primeiro grupo $(28,2 \%)$ e $94(71,8 \%)$ no segundo. Dos pacientes com valores de ISS superiores a 15, $68(72,3 \%)$ apresentaram complicações e 35 foram a óbito $(37,2 \%)$, enquanto que no grupo de pacientes com valores de ISS iguais ou inferiores a 15 houve 17 complicações $(45,9 \%)$ e apenas 1 óbito $(2,7 \%)$. Houve significância estatística quando comparados os grupos tanto com relação à morbidade $(P=$ $0,008)$ quanto à mortalidade $(P<0,0001)$ (Figura 7$)$.



FIGURA 7. ISS relacionado com morbidade e mortalidade (valor de corte do ISS $=15$ )

O ATI variou de 5 a 70, com média de 30. Observaram-se 66 pacientes $(50,4 \%)$ com valor de ATI menor ou igual a 25 e outros 65 com valores superiores a $25(49,6 \%)$. No grupo de pacientes com ATI inferior ou igual a 25 houve 32 casos com complicações $(48,5 \%)$ e 8 óbitos (12,1\%). Dentre os pacientes com valores de ATI superiores a 25 foram encontrados 53 pacientes com complicações pós-operatórias $(81,5 \%)$ e 28 óbitos (43,1\%) (Figura 8).

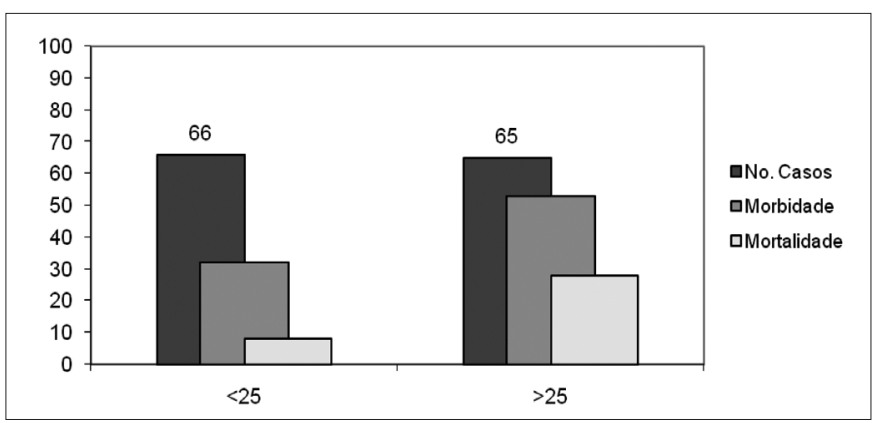

FIGURA 8. ATI relacionado com morbidade e mortalidade

Comparando-se os dois grupos com relação à morbidade e mortalidade, foi encontrada diferença significativa entre eles nos dois casos $(P<0,0001)$.

O TRISS variou de 0,04 a 0,99 , com média de 0,88 , encontrando-se superior a 0,5 em 118 casos $(90,1 \%)$, sendo de 0,99 em 81 pacientes $(61,8 \%)$. Apenas $13(9,9 \%)$ pacientes apresentavam valores de TRISS inferiores a 0,5 e neste grupo todos evoluíram com complicações pós-operatórias e óbito.

No grupo de pacientes com valores de TRISS iguais ou superiores a $0,5,72(61 \%)$ apresentaram complicações pósoperatórias e 23 evoluíram a óbito (19,5\%) (Figura 9).



FIGURA 9. Relação de valor de TRISS com morbidade e mortalidade

Comparando-se os dois grupos observou-se diferença significante entre eles tanto com relação à morbidade $(P<0,003)$ quanto a mortalidade $(P<0,0001)$.

Analisando-se separadamente o grupo submetido a pancreatectomia distal e dividindo-o em dois subgrupos, um com os casos submetidos a esplenectomia e outro com os casos em que houve preservação esplênica, encontrou-se que o grupo em que foi realizada esplenectomia, constituído por 29 pacientes, apresentou ATI média de 35,6, enquanto que no grupo com preservação esplênica, constituído por 
10 pacientes, o ATI médio foi de 20,3. O período médio de internação foi de 13 dias no grupo em que foi realizada esplenectomia e de 18 dias no grupo em que o baço foi preservado. Neste último, a morbidade foi de $70 \%$, com sete pacientes apresentando complicações pós-operatórias, sendo cinco casos de fístula pancreática e dois pacientes que evoluíram para pancreatite aguda pós-operatória. No grupo em que foi realizada esplenectomia associada foram observadas complicações em 22 pacientes, com índice de morbidade de $75,9 \%$. Treze pacientes apresentaram complicações diretamente relacionadas ao procedimento, com nove casos de fístula pancreática e quatro de pancreatite.

A mortalidade dos doentes submetidos a pancreatectomia distal foi de $28,2 \%$, com 11 pacientes indo a óbito, sendo que 2 ocorreram no grupo onde houve preservação esplênica e outros 9 no grupo em que foi realizada esplenectomia associada. Portanto, a mortalidade no primeiro grupo foi de $20 \%$ e no segundo, de $31 \%$. Comparando-se os dois grupos com relação a morbidade e mortalidade, não foi encontrada diferença estatisticamente significante entre eles $(P=0,69)$ (Figura 10).

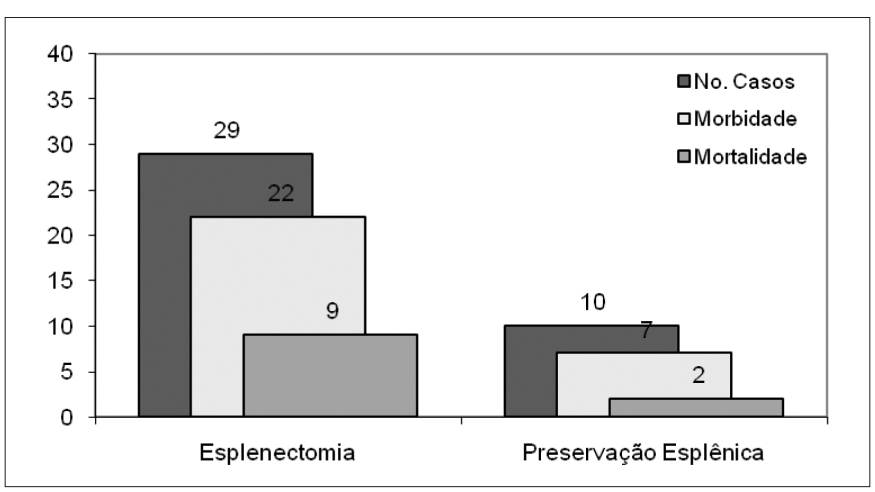

FIGURA 10. Comparação entre grupo submetido a esplenectomia e grupo com preservação esplênica com relação à morbidade e à mortalidade

\section{DISCUSSÃO}

Lesões traumáticas do pâncreas apresentam evolução mais desfavorável quando comparadas às lesões de outros órgãos. Isto ocorre porque, além das complicações diretamente relacionadas à gravidade da lesão, como hemorragia e evolução com formação de fístulas, ainda pode ocorrer quadro de pancreatite aguda, com vasodilatação, choque e aumento do catabolismo, mesmo em lesões menos extensas ${ }^{(27)}$.

No presente estudo foi observado predomínio de pacientes com faixa etária na terceira década de vida, coincidindo com dados da literatura ${ }^{(26)}$, que indicam diminuição da idade das vítimas de trauma abdominal.

Foi encontrado também predomínio do sexo masculino, não havendo relação do sexo com a morbimortalidade.

O trauma penetrante foi encontrado em $64 \%$ dos casos, sendo o agente mais frequente o ferimento por projétil de arma de fogo. Dentre os agentes de trauma contuso, o predominante foi acidente automobilístico. Algumas casuísticas corroboram o predomínio dos traumas penetrantes em jovens e do sexo masculino, com até 94\% dos mesmos apresentando este perfil $^{(2,21)}$

Nos estudos referidos anteriormente houve maior gravidade nos casos de trauma penetrante quando comparados aos casos de trauma fechado. Nesta casuística, embora não tenha sido encontrada diferença significante estatisticamente em relação a estes dois grupos, observou-se tendência a maior morbidade nos pacientes vítimas de traumas penetrantes.

$\mathrm{Na}$ presente série, 17 pacientes com lesões menos graves do pâncreas, graus I e II, apresentaram complicações pósoperatórias, com índice de morbidade de $21 \%$. Neste mesmo grupo ocorreram 17 óbitos, com índice de mortalidade de $21 \%$. Analisando os pacientes com lesão pancreática mais grave, graus IV e V, foram encontrados cinco com complicações pós-operatórias $(35,8 \%)$ e nove óbitos $(64,3 \%)$. Este grupo de pacientes também apresentou valores maiores de ATI, ISS e TRISS, e valores baixos de RTS, corroborando a utilização destes índices como fatores preditivos de mortalidade.

O ATI avaliou a gravidade da lesão pancreática e das de outros órgãos abdominais. Observou-se que valores maiores ou iguais a 25 para este índice indicavam maior probabilidade de óbito, havendo diferença estatística significante, sendo o mesmo observado com relação ao aparecimento de complicações pós-operatórias.

Por se tratar de índice anatômico, avaliando a gravidade da lesão pancreática e de outros órgãos abdominais, mesmo em casos de lesão pancreática menos extensa, com valores menores de ATI, pode haver complicações decorrentes do quadro de pancreatite. Estudo recente com 75 pacientes vítimas de lesão traumática do pâncreas identificou como fator preditivo de morbidade, a acidose metabólica e como fator preditivo de mortalidade, a instabilidade hemodinâmica e choque hemorrágico, porém não foram analisados os índices de trauma ${ }^{(16)}$.

Isto posto, pode-se concluir que o ATI é um índice adequado para indicar probabilidade de mortalidade e sobrevida, bem como de morbidade nos casos de traumatismos pancreáticos.

Além das lesões dos órgãos abdominais, o ISS avalia também os outros segmentos corpóreos. Com relação a este índice observam-se resultados e valores de corte semelhantes aos encontrados no ATI. Pacientes com valores de ISS superiores a 25 apresentam maior probabilidade de óbito, havendo diferença estatística significante entre estes dois grupos $(P=0,003)$. Com relação à morbidade, este valor de corte não a mostrou $(P=0,77)$.

Alterando-se o valor de corte de ISS para 15, foi observada diferença estatística significante tanto para morbidade $(P=$ $0,008)$ quanto para mortalidade $(P<0,001)$. Esta diferença entre os valores de corte do ISS com relação a complicações deve-se ao fato de, em casos de lesão abdominal isolada, como casos de trauma penetrante por arma branca ou projétil de arma de fogo, muitas vezes ser encontrado baixo valor de ISS, porém o paciente evolui com complicações decorrentes da lesão pancreática ou lesões de outros órgãos associadas. 
Estas complicações, entretanto, nem sempre são fatais, com o paciente apresentando sobrevida.

Analisando-se os resultados do presente trabalho, pode-se concluir que o índice anatômico multissegmentar, ISS, em casos de trauma do pâncreas é bom preditor de mortalidade, não se mostrando muito adequado como fator preditivo de morbidade, pois mesmo pacientes com valores baixos, devido à lesão abdominal isolada, podem apresentar complicações.

O grupo de pacientes que apresentou RTS com valor abaixo do máximo, ou seja, valor alterado de RTS, apresentou diferença significativa, quando comparado com o grupo com RTS máximo (normal) tanto para morbidade quanto para mortalidade $(P<0,0001)$.

Sendo o RTS um índice fisiológico, mostrou-se bom como fator preditivo de morbidade e mortalidade. Lesões pancreáticas levam à alteração do RTS devido à queda da pressão arterial sistólica decorrente de hemorragia e choque, bem como da vasodilatação. A frequência respiratória também se altera em casos mais graves devido à hemorragia, como forma de otimizar a oxigenação e também para compensar uma acidose metabólica, com eliminação de gás carbônico (alcalose respiratória). Outros estudos também descreveram aumento da mortalidade em vítimas de trauma abdominal com valores de RTS menores que o valor máximo ${ }^{(9,11)}$.

Considera-se que o RTS é um índice que pode ser considerado fator preditivo tanto de morbidade quanto de mortalidade em casos de traumatismo pancreático.

$\mathrm{Na}$ presente série, observou-se que o grupo de pacientes com valores de TRISS menores ou iguais a 0,5 apresentou maior probabilidade de óbito, quando comparado ao de pacientes com valores de TRISS acima de 0,5 , havendo diferença estatística significante $(P<0,0001)$. A evolução com complicações também apresentou relação com os valores do TRISS. Todos os pacientes com TRISS inferior ou igual a 0,5 evoluíram com complicações e óbito. Este índice foi criado para avaliar probabilidade de sobrevida em pacientes vítimas de trauma e, na presente casuística, foram encontrados $61,8 \%$ dos doentes ( 81 casos) com valor máximo de TRISS, porém a mortalidade observada neste grupo foi maior que a esperada quando comparada ao estudo que definiu este índice ${ }^{(7)}$. Este fato se deve a que doentes jovens, com valores baixos de ISS e RTS alterado, mas com valor alto, próximo de 7 apresentaram valores altos de TRISS, porém evoluíram com complicações e óbito. Pode-se concluir que valores baixo de TRISS são preditores de má evolução em casos de trauma de pâncreas, com complicações pós-operatórias e óbito porém, valores elevados de TRISS não são suficientes para excluir má evolução, podendo o paciente evoluir com complicações e óbito.

Lesões de outros órgãos abdominais associadas ocorreram em 118 pacientes, havendo 39 casos de lesão de grandes vasos abdominais, dos quais 12 de veia cava inferior e 3 de aorta. Estes pacientes apresentavam-se instáveis hemodinamicamente e seis deles foram a óbito durante a cirurgia.

Todos os pacientes vítimas de trauma penetrante foram submetidos a cirurgia precocemente, porém 26 , vítimas de trauma contuso, aguardaram mais de 6 horas para a mesma, sendo que em 9 casos ela foi realizada após 12 horas e em 2 casos após 24 horas do trauma. Estes dois pacientes com diagnóstico tardio da lesão, acima de 24 horas após o trauma, apresentavam quadro de pancreatite aguda, com peritonite e formação de abscesso intracavitário no momento da cirurgia e mesmo com valores elevados de TRISS e ISS evoluíram a óbito. Nestes dois casos, o valor de RTS era alterado e de ATI elevado. Estudos com vítimas de trauma de pâncreas contuso e diagnóstico tardio mostram aumento da morbidade devido à contaminação da cavidade peritonial e formação de abscesso intracavitário ${ }^{(1,2,3)}$.

A escolha do procedimento cirúrgico a ser realizado baseouse no grau e características da lesão pancreática. O grupo de pacientes com lesão grau I submetidos apenas a drenagem apresentou oito casos que evoluíram com complicações pósoperatórias $(15,7 \%)$ e sete que foram a óbito $(13,7 \%)$. Nove pacientes com lesão grau II apresentaram complicações (30\%) pós-operatórias e 10 doentes $(33,3 \%)$ deste grupo evoluíram a óbito, 1 deles durante a cirurgia, decorrente de choque hipovolêmico.

Os pacientes com lesão pancreática distal foram submetidos a pancreatectomia distal com ou sem esplenectomia associada. A mortalidade neste grupo foi de $28,2 \%$, sendo $31 \%$ no grupo em que foi realizada esplenectomia e $20 \%$ no grupo onde o baço foi preservado. A morbidade nestes dois grupos foi de $75,9 \%$ nos casos em que foi realizada esplenectomia e $70 \%$ nos casos em que houve preservação esplênica. Não houve diferença significante entre estes dois grupos com relação à mortalidade ou à morbidade. Era esperado que os pacientes com lesão pancreática distal não envolvendo o baço e que foram submetidos a pancreatectomia distal com preservação esplênica, apresentassem menores índices de complicações e óbito, uma vez que se apresentavam hemodinamicamente estáveis e com melhores valores de índices de trauma como ISS e ATI, à admissão. O RTS neste grupo era normal. A média do ATI foi de 20,3, enquanto que no grupo submetido a esplenectomia havia doentes hemodinamicamente instáveis, com valores de RTS alterados e com média do ATI neste grupo de 35,6. YADAV et al. ${ }^{(29)}$, em estudo que enfatizou a necessidade de se preservar o baço, relata seis pacientes vítimas de lesão pancreática distal traumática submetidos a pancreatectomia distal com preservação esplênica, com um óbito de vítima de choque hemorrágico. O tempo de cirurgia nesta série esteve entre 4 horas e meia e 5 horas. $\mathrm{Na}$ presente casuística, todas as complicações observadas no grupo de pacientes submetidos a pancreatectomia distal com preservação esplênica, foram relacionadas ao procedimento, com cinco casos de fístula pancreática $(50 \%)$ e dois de pancreatite aguda $(20 \%)$. Atribuiu-se o fato de a morbidade e a mortalidade ser a mesma nestes dois grupos a maior tempo cirúrgico, manipulação excessiva do pâncreas e sangramento devido à preservação esplênica. Este procedimento, com preservação esplênica em casos de lesão traumática do pâncreas distal, é controverso, pois pode aumentar o tempo cirúrgico, provocar sangramento e com isso aumentar morbidade e mortalidade. Para a realização deste procedimento é necessário que o cirurgião 
tenha experiência em trauma, de modo a minimizar estas complicações ${ }^{(2,5)}$.

Pacientes submetidos a procedimentos mais complexos, como gastroduodenopancreatectomias, apresentaram maior morbidade e maior mortalidade quando comparados a pacientes submetidos apenas a pancreatectomia distal ou procedimentos de sutura, desbridamento e drenagem, de acordo com outros autores que também relatam maior morbidade e mortalidade em procedimentos complexos ${ }^{(13}$, $18,19,20)$. Pode-se atribuir a isso o fato de que pacientes submetidos a cirurgias mais complexas apresentarem lesão pancreática mais grave e também associação com lesão duodenal, exigindo correção de ambas. Um grupo de pacientes submetidos apenas a drenagem ou sutura da lesão pancreática apresentou evolução desfavorável com complicações e óbito. Deve-se observar que, conforme já descrito, estes pacientes apresentavam piores valores de RTS, ISS e TRISS. Assim, conclui-se que o procedimento cirúrgico realizado, se analisado isoladamente, não é um bom fator preditivo de mortalidade, devendo ser analisado conjuntamente aos índices de trauma.

As complicações pós-operatórias relacionadas diretamente à lesão pancreática e tipo de cirurgia realizada, ocorreram em 38 pacientes e incluíram fístula pancreática, pancreatite aguda, formação de abscesso intracavitário e ressangramento do sítio pancreático, com índice de morbidade de $29 \%$. Estudos referem até $45 \%$ de complicações pós-operatórias diretamente relacionadas à lesão pancreática, tais como fístula pancreática, formação de pseudocistos e necrose tecidual com infecção ${ }^{(1,8,16)}$. Em estudo com 193 pacientes vítimas de trauma pancreático, KAO et al. ${ }^{(20)}$ apresentam maiores índices de complicações relacionadas à lesão em pacientes submetidos a pancreatectomia distal, com lesão grau III do pâncreas, predominando as fístulas pancreáticas. Nesse mesmo trabalho, o ISS, único índice analisado, não apresenta relação com morbidade ou mortalidade, porém 135 doentes apresentavam lesão grau I ou II.

A causa de óbito mais frequente foi choque hemorrágico, decorrente da lesão pancreática e de lesões associadas, e falência de múltiplos órgãos e sistemas, secundária a quadro de reação inflamatória sistêmica, com má perfusão tecidual, insuficiência renal, edema de alças intestinais e dificuldade de troca gasosa pulmonar ${ }^{(3,20)}$. Este quadro clínico, composto pelos fatores descritos anteriormente, corresponde a quadro de pancreatite aguda grave, de etiologia traumática, já descrito para casos de etiologia não-traumática ${ }^{(12)}$.

Coincidentemente com o observado por outros autores ${ }^{8,}$ ${ }^{30)}$, complicações diretamente relacionadas à lesão pancreática como sangramento, foi a principal causa de óbito e a principal complicação relacionada à lesão pancreática e à cirurgia, foi formação de fístula pancreática. Observou-se também parcela significativa de óbitos em decorrência de sepse e falência de múltiplos órgãos e sistemas. Pode-se atribuir isso ao fato de que na lesão pancreática, diferentemente da de outros órgãos, ocorre a liberação de diversas enzimas, peptídios e outros mediadores de vasodilatação e resposta inflamatória, de forma que lesões teciduais pancreáticas acarretam reações sistêmicas de difícil controle, mesmo com a correção cirúrgica da lesão e dos fatores locais ${ }^{(24,25)}$. Desta forma, menor manipulação e ressecção pancreática associada à hemostasia adequada permite minimizar as complicações e melhorar a sobrevida.

Uma vez conhecidos os fatores preditivos de morbidade e mortalidade no trauma pancreático, bem como as particularidades deste tipo de lesão, o cirurgião pode avaliar a probabilidade de o paciente evoluir com complicações pós-operatórias e até mesmo para óbito e assim optar pelo procedimento cirúrgico mais adequado, de modo a minimizar morbidade e mortalidade.

\section{CONCLUSÃO}

A análise dos resultados do presente estudo permite concluir que, no trauma do pâncreas, a morbidade está relacionada a fatores como:

- grau da lesão pancreática, sendo maior em lesões graus IV e V;

- parâmetros fisiológicos como pressão arterial sistêmica e RTS, sendo maior em casos de PAS inferior a $90 \mathrm{~mm} \mathrm{Hg}$ e em casos onde o RTS encontra-se alterado;

- ATI, sendo maior quando o valor do ATI é superior a 25 ;

- ISS, sendo maior quando o valor do ISS é superior a 15.

A mortalidade está relacionada diretamente com todos os índices de trauma estudados, sendo de pior prognóstico quando:

- a lesão pancreática apresenta graus IV ou V;

- a pressão arterial sistêmica é inferior a $90 \mathrm{~mm} \mathrm{Hg}$;

- o RTS apresenta valor alterado;

- o valor do ISS é superior a 25;

- o valor do ATI é superior a 25.

Valores de TRISS inferiores a 0,5 são preditivos de maior morbidade e mortalidade, porém, valores elevados de TRISS não são preditores de boa evolução. 
Silveira HJV, Mantovani M, Fraga GP. Trauma of pancreas: predictor's factors of morbidity and mortality related to trauma index. Arq Gastroenterol. 2009;46(4):270-8.

ABSTRACT - Context - Although relatively uncommon, traumatic pancreatic injury is associated with significant morbidity and mortality. Objective - To define the predictors' factors of increase in the morbidity and mortality in patients with pancreatic trauma. Method - In this casuistic 131 patients were studied, since January 1994 through December 2007, with theirs epidemiological, physiological and anatomic parameters compared and the analysis of the predictive values for the occurrence of bad evolution, with an appropriate statistical study. Results - Penetrating trauma occurred in $64 \%$ and blunt trauma in $36 \%$, and $91.6 \%$ was male. The mean age was 29,8 years. The global morbidity in this series was $64.9 \%$ with $29 \%$ prevalence of pancreas related complications, such as pancreatic fistula and bleeding occurrence. The overall mortality was $27.5 \%$ and occurred by hemorrhagic shock and multiple organs and system failed. Conclusions - Higher morbidity and mortality was related with complex injuries of the pancreas (grade IV and V), but morbidity and mortality in the group of injuries grade I and II are not minimal in patients with changed values of revised trauma score and high values of injury severity score and abdominal trauma index. Systolic blood pressure lower $90 \mathrm{~mm} \mathrm{Hg}$, changed values of revised trauma score index, values of injury severity score higher 15 and values of abdominal trauma index higher 25 are predictive factors of morbidity. Changed values of revised trauma score, values of injury severity score or abdominal trauma index higher 25 , systolic blood pressure are predictive factors of mortality in patients with pancreatic trauma. Low values of TRISS are predictive of higher morbidity and mortality, but high values of TRISS are not predictives of satisfactory evolution.

HEADINGS - Pancreas, injuries. Trauma severity indices.

\section{REFERÊNCIAS}

1. Akhrass R, Yaffe MB, Brandt CP, Reigle M, Fallon WF, Malangoni MA. Pancreatic trauma: a ten year multi-intitutional experience. Am Surg. 1997;63:598-604.

2. Asensio JA, Demetriades D, Hanpeter DE, Gambaro E, Chahwan S. Management of pancreatic injuries. Curr Probl Surg. 1999;36:325-419.

3. Asensio JA, Petrone P, Roldan G, Pak R, Salim A. Pancreatic and duodenal injuries. Complex and lethal. Scand J Surg. 2002;91:81-6.

4. Baker SP, O'Neill B, Haddon W, Long WB. The injury severity score: a method for describing patients with multiples injuries and evaluating emergency care. J Trauma. 1974;14:187-96.

5. Boffard KD, Brooks AJ. Pancreatic trauma-injuries to the pancreas and pancreatic duct. Eur J Surg. 2000;166:4-12.

6. Borlase BC, Moore EE, Moore FA. The abdominal trauma index - a critical reassessment and validation. J Trauma. 1990;30:1340-4.

7. Boyd CR, Tolson MA, Copes WS. Evaluating trauma care: the TRISS method. J Trauma. 1987;27:370-8.

8. Bradley EL, Young PR, Chang MC, Allen JE, Baker CC, Meredith W, Reed L, Thomason M. Diagnosis and initial management of blunt pancreatic trauma: guidelines from a multiinstitution review. Ann Surg. 1998;227:861-9.

9. Champion HR, Sacco WJ, Coppes WS, Gann DS, Gennarelli TA, Flanagan ME. A revision of the trauma score. J Trauma. 1989;29:623-9.

10. Champion HR, Coppes WS, Sacco WJ, Gann DS, Flanagan ME. The major trauma outcome study: establishing national norms for trauma care. J Trauma. 1990;30:1356-65.

11. Coimbra RSM, Angle N, Silva LE, Hoyt DB, Rasslan S. Índices de trauma: o que são e por que devem ser usados? Rev Col Bras Cir. 1997;24:255-63.

12. De Campos T, Cerqueira C, Kuryura L, Parreira JG, Soldá S, Perlingeiro JA, Assef JC, Rasslan S. Morbimortality indicators in severe acute pancreatitis. JOP. 2008;9:690-7.

13. Degiannis E, Glapa M, Loukogeorgakis SP, Smith MD. Management of pancreatic trauma. Injury. 2008;39:21-9.

14. Feliciano DV, Martin TD, Cruse PA, Graham JM, Burch JM, Mattox KL, Bitondo CG, Jordan GL Jr. Management of combined pancreatoduodenal injuries. Ann Surg. 1987;205:673-80.

15. Huerta S, Bui T, Porral D, Lush S, Cinat M. Predictors of morbidity and mortality in patients with traumatic duodenal injuries. Am Surg. 2005;71:763-7.

16. Hwang SY, Choi YC. Prognostic determinant in patients with traumatic pancreatic injuries. J Korean Med Sci. 2008;23:126-30.
17. Jones WG, Reilly DM, Barie PS. Pancreatic injuries. Diagnosis, treatment. AORN J. 1991;53:917-33.

18. Jurczak F, Kahn X, Letessier E, Plattner V, Heloury Y, Le Neel JC. Severe pancreaticoduodenal trauma: review of a serie of 30 patients. Ann Chir. 1999;53:267-72.

19. Jurkovich GJ, Carrico CJ. Pancreatic trauma. Surg Clin N Am. 1990;70:575-93.

20. Kao LS, Bulger EM, Parks DL, Byrd GF, Jurkovich GJ. Predictors of morbidity after traumatic pancreatic injury. J Trauma. 2003;55:898-905.

21. Machado MAC, Volpe P, Souza Jr AL, Poggetti RS, Branco P, Birolini D. Lesões traumáticas do pâncreas: análise de 65 casos. Rev Hosp Clin Fac Med S Paulo. 1994;49:238-42.

22 Moore EE, Dunn EL, Moore JB. Penetrating abdominal trauma index. J Trauma. 1981;21:439-45.

23 Moore EE, Cogbill TH, Malangoni MA, Jurkovich GJ, Champion HR, Gennarelli TA, McAninch JW, Pachter HL, Shackford SR, Trafton PG. Organ Injury Scaling, II: pancreas, duodenum, small bowel, colon, and rectum. J Trauma. 1990;30:1427-9.

24 Patton JH Jr, Fabian TC. Complex pancreatic injury. Surg Clin North Am. 1996;76:783-95.

25 Patton JH Jr, Lyden SP, Croce MA, Pritchard FE, Minard G, Kudsk KA, Fabian TC. Pancreatic trauma: a simplified management guideline. J Trauma. 1997:43:234-9.

26 Rasslan S, Casaroli AA, Abrantes WL, Mantovani M. Pancreatectomia distal no trauma: estudo multicêntrico. Rev Col Bras Cir. 1999;25:409-14.

27 Subramanian A, Dente CJ, Feliciano DV. The management of pancreatic trauma in the modern era. Surg Clin N Am. 2007;87:1515-32.

28 Vasquez JC, Coimbra R, Hoyt DB, Fortlage D. Management of penetrating pancreatic trauma: an 11 year experience of a level 1 trauma center. Injury. 2001;32:753-9

29 Yadav TD, Natarajan SK, Kishore VM, Lyngdoh S, Wig JD. Spleenpreservating distal pancreatectomy for pancreatic trauma: a serie of six cases. JOP. 2007;8:422-8.

30 Young PR, Meredith JW, Baker CC. Pancreatic injuries resulting from penetrating trauma: a multi-institution review. Am Surg 1998;64:838-44. 